\title{
Estimativa de contaminação de águas subterrâneas e superficiais por agrotóxicos em área sucroalcooleira, Santa Rita/PB, Brasil
}

\author{
Estimate of the contamination of groundwater and surface water \\ due to agrochemicals in the sugar-alcohol area, Santa Rita, State of \\ Paraíba, Brazil
}

Luara Lourenço Ismael (https://orcid.org/0000-0002-8773-8256) ${ }^{1}$

Elisângela Maria Rodrigues Rocha (https://orcid.org/0000-0001-7024-6979) ${ }^{2}$
${ }^{1}$ Programa de PósGraduação em Engenharia Civil e Ambiental, Centro de Tecnologia, Universidade Federal da Paraíba (UFPB).

Campus I, Cidade Universitária. 58059-900 João Pessoa PB Brasil. luara_ismael@hotmail.com ${ }^{2}$ Departamento de Engenharia Civil e Ambiental, Centro de Tecnologia, UFPB. João Pessoa PB Brasil.

\begin{abstract}
The increase in agricultural productivity, especially sugarcane production, is generally associated with the use of agrochemicals. The risk of contamination of these compounds in water resources is a concern for public and environmental health. In this context, the scope of this study was to estimate the contamination potential of the groundwater and surface water by agrochemicals applied in a sugar and alcohol area in the metropolitan region of João Pessoa, Santa Rita/Paraíba. Analysis was performed according to the criteria proposed by the EPA (Environmental Protection Agency), namely the GUS index (Groundwater Ubiquity Score) and the GOSS method (surface water). As a result of the estimate of groundwater contamination, it was observed that $52 \%$ out of 25 active compounds evaluated by the methods (EPA and GUS), showed a tendency of potential contamination. With respect to the contamination of surface waters, most of the active principles had a greater tendency to transport their molecules dissolved in water and transported them through rainwater. Thus, the conclusion drawn is that there is a need for the implementation of environmental monitoring programs and measures aimed at the protection of public health.
\end{abstract}

Key words Agrochemicals, Water resources, Environment, Public health
Resumo O aumento da produtividade agrícola, em especial da produção canavieira, geralmente está associado ao uso de agrotóxicos. O risco de contaminação desses compostos nos recursos hídricos é uma preocupação para a saúde pública e ambiental. Nesse contexto, o estudo objetivou estimar o potencial de contaminação das águas subterrâneas e superficiais por agrotóxicos aplicados em uma área sucroalcooleira na região metropolitana de João Pessoa, município de Santa Rital Paraíba. A análise foi realizada seguindo critérios propostos pela Environmental Protection Agency EPA e indice de GUS (águas subterrâneas) e pelo método de GOSS (águas superficiais). Como resultados da estimativa de contaminação das águas subterrâneas, observou-se que dos 25 princípios ativos avaliados pelos métodos (EPA e GUS), 52\% apresentaram tendência de contaminação potencial. Quanto ao risco de contaminação das águas superficiais, a maioria dos princípios ativos possuía uma maior tendência de transporte de suas moléculas dissolvidas em água e transportadas por meio do carreamento das águas da chuva. Assim, pode-se concluir que há a necessidade de implantação de programas de monitoramento ambiental e de medidas voltadas à proteção da saúde pública.

Palavras-chave Agrotóxicos, Recursos hídricos, Meio ambiente, Saúde pública. 


\section{Introdução}

A busca por maior produtividade agrícola resulta no uso de agrotóxicos, que tem se intensificado consideravelmente ${ }^{1,2}$. Destacam-se as grandes monoculturas como a cana-de-açúcar, que é considerada uma das culturas de maior consumo de agrotóxicos no Brasil ${ }^{3-5}$. No estado da Paraíba, a expansão da monocultura da cana-de-açúcar vem acompanhada de modificações significativas, que resultaram em degradação ambiental culminando na contaminação dos recursos hídricos e do solo por agrotóxicos, e contribuindo para um desequilíbrio de toda a biodiversidade ${ }^{6,7}$. $\mathrm{Na}$ concepção de Figueiredo ${ }^{8}$ a maioria das usinas e destilarias utilizam em seu ciclo produtivo práticas degradantes ao meio ambiente e à saúde da população.

Dados do Sistema Nacional de Informações Tóxico-Farmacológicas - SINITOX, gerenciado pela Fundação Oswaldo Cruz - Fiocruz, informam que no estado da Paraíba entre os anos de 2006 a 2009 e 2011 a 2012, foram registrados 635 casos de intoxicações e 40 casos de morte por agrotóxicos de uso agrícola. $\mathrm{O}$ ano de 2007 destacou-se por apresentar o maior número de casos de intoxicação (178) e morte (13), enquanto que no ano de 2012 contatou-se os menores números de casos?.

O impacto direto da contaminação humana por agrotóxicos é percebido por meio de três vias principais: ocupacional, ambiental e alimentar. A via ambiental caracteriza-se pela dispersão e dinâmica dos resíduos de agrotóxicos nos diferentes compartimentos ambientais ${ }^{10}$. A ingestão diária de água e de alimentos contaminados por agrotóxicos pode ocasionar o acúmulo dessas substâncias no organismo humano, podendo causar graves doenças ${ }^{11}$. Arias, et al. ${ }^{12}$ comentam que quanto maior for a concentração de pesticidas e mais longo for o tempo de exposição, maiores serão as chances de ocorrência dos impactos negativos atingirem níveis superiores de organização biológica, como comunidades e ecossistemas.

Os danos dos agrotóxicos à saúde humana podem ser observados em estudos de carcinogênese, mutagênese, teratogênese, neurotoxicidade, alterações imunológicas e na reprodução de animais, desregulações endócrinas, alterações no desenvolvimento embrio-fetal, após exposição in útero, com efeitos capazes de acarretar a morte dos indivíduos, comprometimento de espécies, mudança da dinâmica bioquímica natural e na mudança do funcionamento do ambiente afeta$\mathrm{do}^{5,13}$.
Alguns tipos de agrotóxicos, ao permanecerem no ambiente ou alcançarem o meio aquático, oferecem riscos para espécies animais por sua toxicidade e possibilidade de bioacumulação na cadeia alimentar ${ }^{14-17}$. O que torna os efeitos ecotoxicológicos e toxicológicos pouco conhecidos ao longo do tempo ${ }^{15}$.

A contaminação dos recursos hídricos por resíduos de agrotóxicos é capaz de provocar sérios problemas à saúde pública, principalmente quando este recurso é utilizado para o abastecimento humano. A presença desses compostos nos mananciais pode acarretar problemas para $\mathrm{o}$ tratamento da água em virtude da eventual necessidade de tecnologias mais complexas do que aquelas normalmente usadas para alcançar a potabilização do recurso ${ }^{18}$.

$\mathrm{Na}$ Portaria do Ministério da Saúde $\mathrm{n}^{\circ}$ 2914/2011 encontra-se regulamentado 64 substâncias químicas que representam riscos à saúde humana, dentre as quais 27 são agrotóxicos, o que de acordo com a Associação Brasileira de Saúde Coletiva - ABRASCO, este número representa menos de $10 \%$ dos princípios ativos oficialmente registrados no País ${ }^{1,19,20}$. Diante dessas informações, a identificação e avaliação de riscos à saúde e ao ambiente se tornam importantes ferramentas para contribuir com o controle e prevenção da exposição da população aos agrotóxicos.

A estimativa do risco de contaminação das águas superficiais e subterrâneas por agrotóxicos vem sendo objeto de estudo de diversas pesquisas. O uso de modelos matemáticos são frequentemente utilizados para estimar o potencial de contaminação. Destaca-se entre estes modelos o método de Screening da Agência de Proteção Ambiental dos Estados Unidos-USEPA e o índice de GUS, ambos capazes de estimar a contaminação para águas subterrâneas e o método de GOSS (para águas superficiais) $)^{15,21-24}$. No entanto, para a aplicação dessas técnicas, é necessário o conhecimento das propriedades físico-químicas dos princípios ativos presentes nas formulações dos agrotóxicos, as quais são capazes de influenciar diretamente no transporte dos resíduos no ambiente.

Sendo assim, este estudo buscou estimar o potencial de contaminação das águas subterrâneas e superficiais, decorrentes do uso de agrotóxicos aplicados na cultura de cana-de-açúcar em uma usina sucroalcooleira localizada na região metropolitana de João Pessoa-Paraíba por meio da aplicação de modelos que analisam as propriedades físico-químicas dos princípios ativos de cada agrotóxico e seu potencial de contaminação ambiental e de saúde pública. 


\section{Métodos}

A presente pesquisa utilizou o método de abordagem descritivo e de finalidade aplicada, a qual objetiva gerar conhecimentos com base em observações, levantamentos documentais e aplicações práticas ${ }^{25,26}$. Na concepção de $\mathrm{Gil}^{27}$, do ponto de vista dos procedimentos técnicos, a pesquisa caracteriza-se como exploratória, adotando-se como método de investigação científica o Estudo de Caso.

\section{Local de estudo}

A Usina Sucroalcooleira estudada localiza-se na Região metropolitana de João Pessoa-PB, entre as cidades de Santa Rita e Espírito Santo, mais precisamente no Engenho Central em Santa Rita -PB. A Usina possui uma área de 19.000 hectares, dos quais 9.000 ha estão direcionados ao cultivo da monocultura da cana-de-açúcar.

O fornecimento hídrico para irrigação da cultura de cana-de-açúcar na usina é assegurado pelos açudes Cafundó e Estivas, ambos, distantes 500 metros da área plantada e $4 \mathrm{~km}$ entre os mesmos. Além destes, pode-se citar o rio Paraíba que é utilizado para fins de irrigação em épocas de escassez hídrica. O fornecimento de água para a atividade industrial é obtido do açude de Reis que localiza-se a 500 metros da área plantada. Para o abastecimento humano, a usina possui dentro das instalações fabris 2 (dois) poços, um em funcionamento e o outro com uso apenas em casos de manutenção.

O manejo dos agrotóxicos na área de estudo é feito durante todo o ciclo vegetativo na cultura de cana-de-açúcar seja nas épocas chuvosas ou nas épocas secas. As aplicações dos agrotóxicos na usina são feitas manualmente pelos aplicadores utilizando pulverizadores costais, mecanizada com o auxílio de pulverizadores mecânicos e por via aérea de forma simultânea nas diferentes fazendas.

\section{Levantamento dos agrotóxicos utilizados no ciclo da cana-de-açúcar da usina}

Os princípios ativos presentes nas marcas comerciais dos agrotóxicos utilizados no processo de produção agrícola canavieiro da usina, foram identificados a partir de visitas técnicas in loco, consulta à bula dos produtos fornecidos pela Agência de Defesa Agropecuária do Paraná-A-
DAPAR $^{28}$ e à base de dados disponibilizadas nas monografias de agrotóxicos publicadas no Portal ANVISA e em suas Resoluções ${ }^{29}$.

Em seguida, foram levantadas informações sobre as seguintes propriedades físico-químicas dos princípios ativos: Solubilidade em água a $20^{\circ} \mathrm{C}(\mathrm{S})$; Coeficiente de adsorção à matéria orgânica do solo (KOC); Constante de Henry $\left(\mathrm{K}_{\mathrm{H}}\right)$; Coeficiente de partição octanol/água $\mathrm{pH}=$ $7,20^{\circ} \mathrm{C}($ Kow $)$; Constante de dissociação à $25^{\circ} \mathrm{C}$ (Pka); Tempo de meia-vida no solo $\left(\mathrm{DT}_{50 \text { solo }}\right)$ e na água $\left(\mathrm{DT}_{50 \text { água }}\right)$ e Pressão de vapor a $25^{\circ} \mathrm{C}(\mathrm{PV})$. As respectivas propriedades físico-químicas dos pesticidas foram obtidas a partir de dados disponibilizados pela ANVISA e do Purchasing the database- $\mathrm{PPDB}^{30}$.

\section{Avaliação do potencial de contaminação de águas subterrâneas}

A estimativa do risco potencial de contaminação de águas subterrâneas na respectiva área de estudo, foi realizada com base em duas metodologias: Critério da Agência de Proteção Ambiental dos Estados Unidos (EPA) e Índice de Groundwater Ubiquity Score (GUS) $)^{14-17}$.

\section{Critério da Agência de Proteção Ambiental dos Estados Unidos (EPA)}

O método de screening sugerido pela EPA na análise preliminar de riscos de contaminação de águas subterrâneas por agrotóxicos considera os seguintes critérios:

- Solubilidade dos pesticidas em água $(\mathrm{S})>$ $30 \mathrm{mg} . \mathrm{L}^{-1}$

- Coeficiente de adsorção à matéria orgânica do solo $(\mathrm{Koc})<300-500 \mathrm{~mL} \cdot \mathrm{g}^{-1}$

- Constante da Lei de Henry $\left(\mathrm{K}_{\mathrm{H}}\right)<10^{-2}$ Pa.m ${ }^{3} \cdot \mathrm{mol}^{-1}$

- Especiação (Esp): negativamente carregado a pH normal do ambiente ( 5 a 8 )

- Meia-vida no solo $>14$ a 21 dias

- Meia-vida na água > 175 dias

Com base nestas propriedades, os princípios ativos que se enquadrem na maioria dos critérios supracitados $(>3)$ indica maior potencial de risco de transporte, e consequentemente foram considerados de alto potencial contaminante das águas subterrâneas. Ao atender apenas 3 critérios foram considerados com o potencial de contaminação intermediário, e abaixo de 3 critérios, não foram considerados contaminantes ${ }^{15,16,31}$. 


\section{Índice de vulnerabilidade de águas subterrâneas (GUS)}

Esse critério foi elaborado por Gustafson ${ }^{32}$, sendo calculado por meio dos valores de meiavida do composto no solo (DT50 ${ }_{\text {solo }}$ ) e do coeficiente de adsorção à matéria orgânica do solo (Koc).

$$
\text { GUS }=\log \left(\text { DT50 }_{\text {solo }}\right) \times(4-\log \text { Koc }) \text { Equação } 1
$$

As faixas de classificação dos compostos de acordo com sua tendência à lixiviação são:

- $\quad$ GUS < 1,8: não sofre lixiviação;

- $\quad 1,8<$ GUS $<2,8$ : faixa de transição;

- $\quad$ GUS $>2,8$ : provável lixiviação.

Além da análise individual dos critérios que estimam a contaminação das águas subterrâneas (EPA e GUS), foi realizada uma avaliação geral do risco de contaminação dos princípios ativos, como proposto no estudo realizado por Milhome et al. ${ }^{15}$ os quais consideram associações com base na análise dos resultados de ambos os métodos.

\section{Avaliação do potencial de contaminação de águas superficiais}

Para análise do risco de contaminação de águas superficiais, foi utilizado o método proposto por $\mathrm{Goss}^{33}$, o qual reúne um conjunto de critérios apresentados em intervalos matemáticos, pelos quais os princípios ativos são classificados em alto e baixo potencial de contaminação em função da capacidade de transporte associado ao sedimento ou dissolvido em águas superficiais (Tabela 1).

A classificação dos princípios ativos em alto e baixo potencial de contaminação foi avaliada com base no atendimento total aos intervalos matemáticos dos três critérios avaliados (DT50; Koc e S). Esta análise foi estabelecida para os dois meios de transportes dos compostos em águas superficiais (associados ao sedimento e dissolvido em água).

Além de serem classificados em alto e baixo potencial de contaminação das águas superficiais, alguns princípios ativos classificam-se como de médio potencial, correspondendo aos agrotóxicos que não se enquadram totalmente em nenhum dos critérios acima ${ }^{31,34}$, ou seja, aqueles princípios ativos em que suas propriedades atendem parcialmente aos intervalos matemáticos estabelecidos por ambos os níveis (alto e baixo potencial).

\section{Resultados e discussões}

\section{Levantamento dos agrotóxicos utilizados no ciclo da cana-de-açúcar da usina}

De acordo com o levantamento do histórico do uso dos agrotóxicos aplicados na cultura de cana-de-açúcar da usina em estudo, utilizavamse um total de 25 princípios ativos (PA) distribuídos em diferentes marcas comerciais (Tabela 2). Todos os princípios ativos em uso encontravamse autorizados para a cultura na Agência Nacional de Vigilância Sanitária- Anvisa ou no Ministério da Agricultura Pecuária e Abastecimento-MAPA.

\section{Avaliação do potencial de contaminação de águas subterrâneas}

O potencial de contaminação de águas subterrâneas utilizando o método da Agência de Proteção Ambiental dos Estados Unidos - EPA e o índice de GUS, para os princípios ativos identificados na área de estudo estão descritos na Tabela 3 .

De acordo com os critérios da EPA, princípios ativos que possuem propriedades, como elevada solubilidade em água, baixa adsorção à matéria orgânica no solo e alta meia-vida no solo, tendem a atingirem o lençol freático, quando comparados com os demais, potencializando a contaminação das águas subterrâneas ${ }^{22,23,35}$. Considera-se como contaminantes potenciais (CP) de acordo com o critério EPA, aqueles princípios ativos em que a maioria $(>3)$ de suas propriedades físicoquímicas atendaram as condições propostas pelo método ${ }^{18-23}$.

Dentre os princípios ativos avaliados quanto ao método EPA, 52\% (Tabela 3), encontram-se inseridos na faixa de potencial contaminação (PC) das águas subterrâneas. Os princípios ativos MSMA, Paraquate, Imazapique e Amicarbazona foram classificados como contaminantes de intermediário potencial de contaminação (IN), enquanto que os compostos: Fipronil, Fluazifope-P, Lambda-Cialotrina, Etefom, Imazapir, Oxyfluorfem e Isoxaflutol, correspondentes a $32 \%$ dos compostos avaliados, classificaram-se como não contaminantes (NC).

Britto $^{16}$, ao avaliar o potencial de contaminação das águas subterrâneas por agrotóxicos aplicados na cultura de cana-de-açúcar na sub-bacia do rio Poxim-SE, observou para a aplicação do método da EPA que os princípios ativos: Metribuzim e Diuron foram classificados como con- 
Tabela 1. Método proposto por GOSS para avaliação do potencial de contaminação de águas superficiais por princípios ativos dos agrotóxicos.

\begin{tabular}{|c|c|c|c|}
\hline & \multicolumn{3}{|c|}{ Potencial de transporte associado ao sedimento } \\
\hline & DT50 $_{\text {solo }}(\mathrm{d})$ & $\mathrm{K}_{o c}\left(\mathrm{~mL} \cdot \mathrm{g}^{-1}\right)$ & S (mg.L-1) \\
\hline \multirow[t]{2}{*}{ Alto Potencial } & $\geq 40$ & $\geq 1000$ & - \\
\hline & $\geq 40$ & $\geq 500$ & $\leq 0,5$ \\
\hline \multirow[t]{5}{*}{ Baixo Potencial } & $>1$ & - & - \\
\hline & $\leq 2$ & $\leq 500$ & - \\
\hline & $\leq 4$ & $\leq 900$ & $\geq 0,5$ \\
\hline & $\leq 40$ & $\leq 500$ & $\geq 0,5$ \\
\hline & $\leq 40$ & $\leq 900$ & $\geq 2$ \\
\hline \multicolumn{4}{|c|}{ Potencial de transporte dissolvido em água } \\
\hline \multirow{3}{*}{ Alto Potencial } & $\mathrm{DT} \mathbf{s o l o}_{\text {so }}(\mathrm{d})$ & $\mathrm{K}_{\mathrm{oc}}\left(\mathrm{mL} \cdot \mathrm{g}^{-1}\right)$ & $\mathrm{S}\left(\mathbf{m g} \cdot \mathrm{L}^{-1}\right)$ \\
\hline & $>35$ & $<100000$ & $\geq 1$ \\
\hline & $<35$ & $\leq 700$ & $\geq 10 \mathrm{e} \leq 100$ \\
\hline \multirow[t]{3}{*}{ Baixo Potencial } & - & $\geq 100000$ & - \\
\hline & $\leq 1$ & $\geq 1000$ & - \\
\hline & $<35$ & - & $<0,5$ \\
\hline
\end{tabular}

Fonte: Milhome (2011) apud Goss ${ }^{33}$.

DT50: meia-vida no solo; Koc: coeficiente de adsorção à matéria orgânica do solo; S: solubilidade em água.

taminantes potenciais, resultado que corrobora com o obtido no presente estudo. Os resultados obtidos por Milhome et al. ${ }^{15}$ evidenciam que a maioria dos compostos analisados pelo método EPA, totalizando 47\%, foram classificados como contaminantes potenciais (CP).

$\mathrm{O}$ índice de GUS enquadra os princípios ativos na condição de provável lixiviação (PL) e faixa de transição (FT), os quais podem oferecer risco potencial de contaminação e os compostos com potencial de lixiviação nulo (LN), ou seja, aqueles considerados não contaminantes de águas subterrâneas ${ }^{34}$. Os compostos que apresentaram índice de GUS na faixa de transição e potencial contaminação quando avaliados pelo critério EPA, também oferecem risco potencial de contaminação das águas subterrâneas ${ }^{34}$.

Conforme mencionado por Silva ${ }^{14}$ e Ruy e Reis $^{36}$ quanto maior a meia-vida do composto e menor absorção da molécula nas partículas de matéria orgânica do solo, maior será o valor de GUS, ou seja, maior seu potencial de lixiviação no perfil do solo e, consequentemente, contaminação das águas subterrâneas.

Dos princípios ativos avaliados, 39\% possuíam tendência de provável lixiviação (PL), das águas subterrâneas, sendo estes: SulfometuromMetílico, Clomazone, Imazapique Tebutiurom, Sulfentrazona, Hexazinona, Picloram, Amicarbazona e Imidacloprido (Figura 1). Os princípios ativos Sulfentrazona $(6,47)$, Picloram $(5,54)$ e Te- butiurom $(5,46)$ destacaram-se, pois obtiveram os maiores valores. Os compostos: Ametrina, MSMA, Fipronil, 2,4 D, Diurom e Metribuzim por estarem inseridos na faixa de transição $1,8<$ GUS > 2,8 também apresentaram tendência de provável lixiviação (PL), os demais (35\%) possuem lixiviação nula (LN).

No estudo desenvolvido por Armas e Monteiro ${ }^{21}$, referente à análise do risco de contaminação das águas subterrâneas (método de GUS) em agrotóxicos utilizados na cultura de cana-de -açúcar na sub-bacia hidrográfica do rio Corumbataí, foi observado uma semelhança aos resultados encontrados na presente pesquisa, onde, a maioria dos princípios ativos avaliados possuíam tendência de provável lixiviação (75\%), além disso, os três compostos que apresentaram a maior tendência de lixiviação para as águas subterrâneas encontrados pelos autores também foram os mesmos diagnosticados neste estudo.

O resultado geral do risco de contaminação das águas subterrâneas (EPA e GUS), mostrou que 13 dos princípios ativos identificados na usina, correspondendo a 52\%, classificaram-se como contaminantes potenciais (CP), 12\% enquadraram-se na classe intermediária de contaminação (IN), 28\% do total classificaram-se como não contaminantes (NC) e o restante por falta de dados das propriedades físico-químicas não foram analisados, sendo, portanto, inconclusivos (I). Tais resultados merecem atenção 


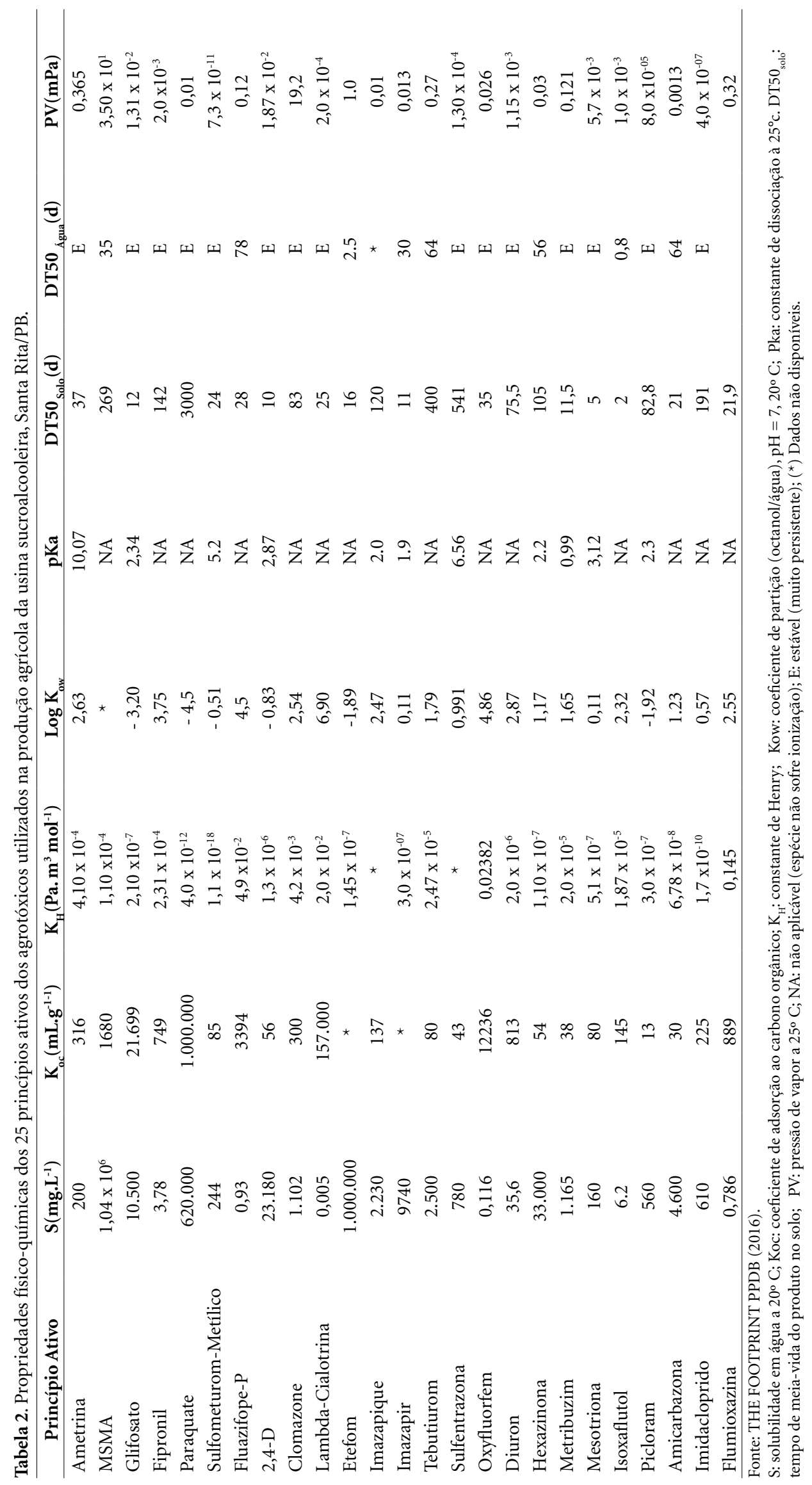


Tabela 3. Avaliação geral do potencial de contaminação de águas subterrâneas pelos 25 princípios ativos utilizados na usina com base nos método da EPA e o Índice de GUS.

\begin{tabular}{|c|c|c|c|c|c|c|c|c|c|c|}
\hline & \multicolumn{6}{|c|}{ Princípio Ativo } & \multirow{2}{*}{$\begin{array}{c}\begin{array}{c}\text { Critério } \\
\text { EPA }\end{array} \\
\text { Análise } \\
\text { EPA }\end{array}$} & \multicolumn{2}{|c|}{ Critério GUS } & \multirow[b]{2}{*}{$\begin{array}{c}\text { Risco } \\
\text { em Água } \\
\text { Subterrânea }\end{array}$} \\
\hline & $S$ & $\mathrm{~K}_{\mathrm{oc}}$ & $\mathbf{K}_{\mathrm{H}}$ & $\mathrm{pKa}$ & $\begin{array}{c}\text { DT50 } \\
\text { soL }\end{array}$ & $\begin{array}{c}\text { DT50 } \\
\text { ÁGU }\end{array}$ & & $\begin{array}{c}\text { Índice } \\
\text { GUS }\end{array}$ & $\begin{array}{c}\text { Análise } \\
\text { GUS }\end{array}$ & \\
\hline Ametrina & $\bullet$ & O & $\bullet$ & O & $\bullet$ & $\bullet$ & $\mathrm{CP}$ & 2,35 & FT & $\mathrm{CP}$ \\
\hline MSMA & $\bullet$ & O & $\bullet$ & O & $\bullet$ & O & IN & 1,88 & FT & IN \\
\hline Glifosato & $\bullet$ & O & $\bullet$ & $\bullet$ & & $\bullet$ & $\mathrm{CP}$ & $-0,36$ & $\mathrm{LN}$ & IN \\
\hline Fipronil & O & O & $\bullet$ & O & O & $\bullet$ & $\mathrm{NC}$ & 2,42 & $\mathrm{FT}$ & $\mathrm{NC}$ \\
\hline Paraquate & $\bullet$ & O & $\bullet$ & O & O & $\bullet$ & IN & $-6,95$ & $\mathrm{LN}$ & $\mathrm{NC}$ \\
\hline Sulfometurom-Metílico & $\bullet$ & $\bullet$ & $\bullet$ & & $\bullet$ & $\bullet$ & $\mathrm{CP}$ & 2,86 & PL & $\mathrm{CP}$ \\
\hline Fluazifope-P & O & O & O & O & $\bullet$ & O & $\mathrm{NC}$ & 0,68 & $\mathrm{LN}$ & $\mathrm{NC}$ \\
\hline $2,4-\mathrm{D}$ & $\bullet$ & $\bullet$ & $\bullet$ & $\bullet$ & O & $\bullet$ & $\mathrm{CP}$ & 2,25 & FT & $\mathrm{CP}$ \\
\hline Clomazone & $\bullet$ & O & $\bullet$ & O & $\bullet$ & $\bullet$ & $\mathrm{CP}$ & 2,92 & PL & $\mathrm{CP}$ \\
\hline Lambda-Cialotrina & O & O & O & O & $\bullet$ & $\bullet$ & $\mathrm{NC}$ & $-1,67$ & $\mathrm{LN}$ & $\mathrm{NC}$ \\
\hline Etefom & $\bullet$ & - & $\bullet$ & O & O & O & NC & - & I & I \\
\hline Imazapique & $\bullet$ & $\bullet$ & - & O & $\bullet$ & - & IN & 3,88 & PL & $\mathrm{CP}$ \\
\hline Imazapir & $\bullet$ & - & O & $\bullet$ & O & O & $\mathrm{NC}$ & - & I & I \\
\hline Tebutiurom & $\bullet$ & $\bullet$ & $\bullet$ & O & $\bullet$ & O & $\mathrm{CP}$ & 5,46 & PL & $\mathrm{CP}$ \\
\hline Sulfentrazona & $\bullet$ & $\bullet$ & - & O & $\bullet$ & $\bullet$ & $\mathrm{CP}$ & 6,47 & PL & $\mathrm{CP}$ \\
\hline Oxyfluorfem & O & O & O & O & $\bullet$ & $\bullet$ & $\mathrm{NC}$ & $-0,14$ & $\mathrm{LN}$ & $\mathrm{NC}$ \\
\hline Diuron & $\bullet$ & $\bullet$ & 0 & $\bullet$ & O & $\bullet$ & $\mathrm{CP}$ & 2,05 & FT & $\mathrm{CP}$ \\
\hline Hexazinona & $\bullet$ & $\bullet$ & $\bullet$ & $\bullet$ & $\bullet$ & O & $\mathrm{CP}$ & 4,58 & PL & $\mathrm{CP}$ \\
\hline Metribuzim & $\bullet$ & $\bullet$ & - & $\bullet$ & O & $\bullet$ & $\mathrm{CP}$ & 2,57 & FT & $\mathrm{CP}$ \\
\hline Mesotriona & $\bullet$ & $\bullet$ & $\bullet$ & $\bullet$ & O & $\bullet$ & $\mathrm{CP}$ & 1,47 & $\mathrm{LN}$ & IN \\
\hline Isoxaflutol & O & $\bullet$ & $\bullet$ & O & O & O & $\mathrm{NC}$ & 0,55 & $\mathrm{LN}$ & $\mathrm{NC}$ \\
\hline Picloram & $\bullet$ & $\bullet$ & • & $\bullet$ & $\bullet$ & $\bullet$ & $\mathrm{CP}$ & 5,54 & PL & $\mathrm{CP}$ \\
\hline Amicarbazona & • & $\bullet$ & $\bullet$ & O & O & O & $\mathrm{IN}$ & 3,34 & PL & $\mathrm{CP}$ \\
\hline Imidacloprido & $\bullet$ & $\bullet$ & $\bullet$ & O & $\bullet$ & $\bullet$ & $\mathrm{CP}$ & 3,76 & PL & $\mathrm{CP}$ \\
\hline Flumioxazina & 0 & O & 0 & O & $\bullet$ & 0 & $\mathrm{NC}$ & 1,41 & $\mathrm{LN}$ & $\mathrm{NC}$ \\
\hline
\end{tabular}

- Atende ao critério EPA; $O$ Não atende ao critério EPA; CP: Contaminante em Potencial;

IN: Intermediário Potencial de Contaminação; NC: Não Contaminante; - (dados não disponíveis); PL: Provável Lixiviação FT:

Faixa de Transição; LN: Lixiviação Nula; I: (Inconclusivo/dados não disponíveis).

quanto ao uso indiscriminado desses compostos no ambiente, principalmente quanto à contaminação das águas subterrâneas, sendo estas, ainda pouco monitoradas nos estudos ambientais.

Quando comparados o método EPA e o Índice de GUS, observou-se que sete princípios ativos (Sulfometurom-Metílico, Clomazone, Tebutiurom, Sulfentrazona, Hexazinona, Picloram, e Imidacloprido) foram classificados como contaminantes potenciais (CP) e que possuíam provável lixiviação (PL). Tais compostos mostraram concordância quando avaliados pelos dois métodos, ou seja, foram os mais suscetíveis de contaminação de águas subterrâneas.

Conforme mencionado por Primel et $\mathrm{al}^{37}$, a análise do potencial de lixiviação dos compostos de acordo com os critérios propostos, apesar de abordar apenas algumas propriedades físico-químicas dos agrotóxicos sem levar em consideração as características do solo e clima, pode ser considerada como ferramenta para a avaliação inicial do potencial de poluição ambiental. A aplicação de altas doses, associado ao alto potencial de lixiviação e solos com baixa capacidade de retenção sugerem uma situação de alto risco dos compostos para o ambiente e para a saúde.

\section{Avaliação do potencial de contaminação de águas superficiais}

A estimativa de contaminação das águas superficiais por meio do transporte das moléculas 


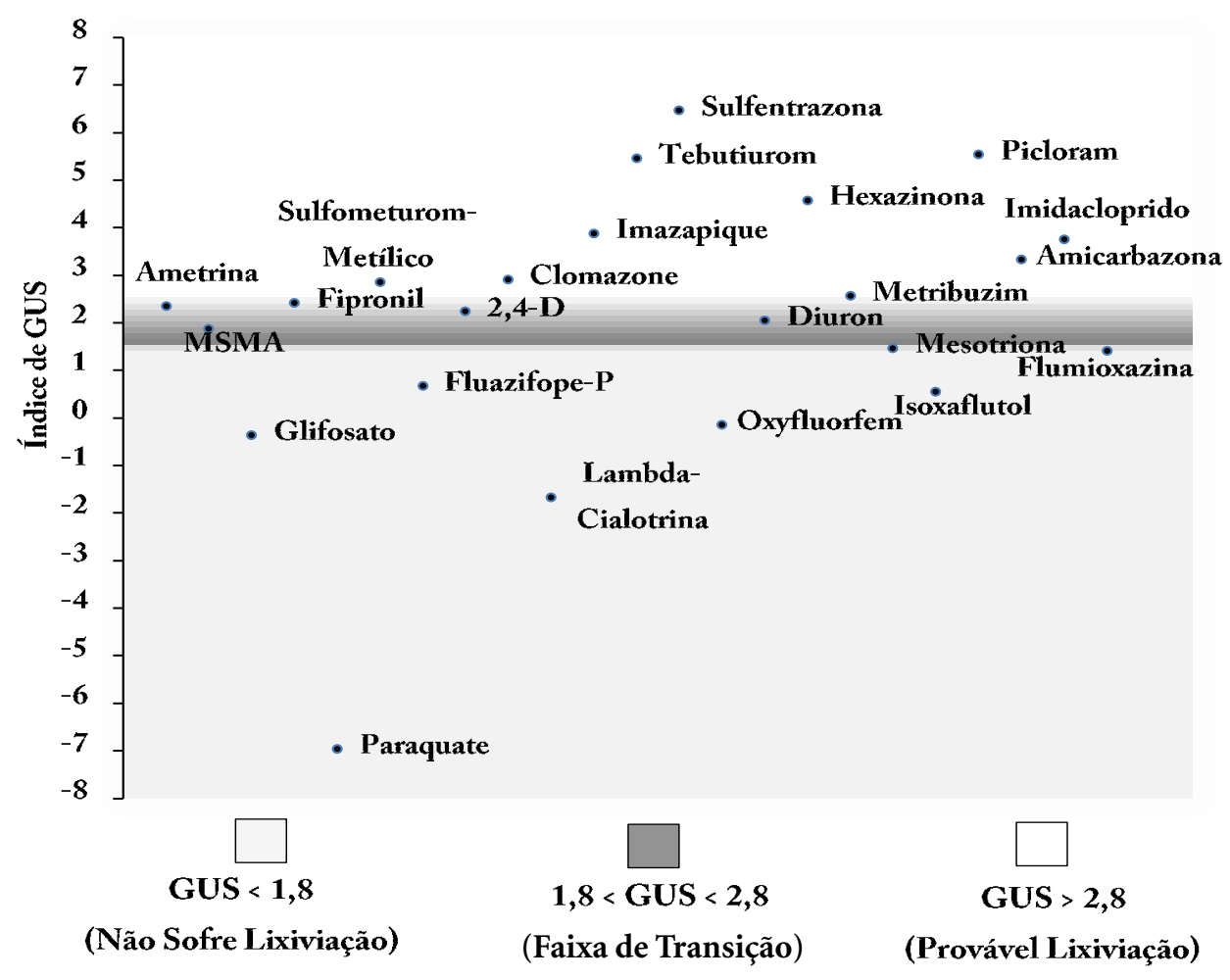

Figura 1. Avaliação do risco de contaminação das águas subterrâneas pelo Índice de GUS para área sucroalcooleira, Santa Rita/PB.

Fonte: Autoras (2016).

associado ao sedimento (PTAS) revelou que 02 compostos $(9 \%)$ possuíam tendência de alto potencial (AP) de contaminação, 14 compostos (61\%) encontravam-se inseridos na faixa de médio potencial (MP) e 07 princípios ativos (30\%), apresentaram tendência de baixo potencial (BP), conforme o Quadro 1. Ressalta-se que foram analisados 23 princípios ativos, sendo excluídos os compostos: Etefom e Imazapir, devido não possuírem informação do valor Koc.

Quando avaliada a contaminação das águas superficiais por meio do transporte dos princípios ativos dissolvidos em água (PTDA), 52\% dos compostos identificados apresentaram tendência de alto potencial (AP) de contaminação; 09 compostos (39\%) foram classificados como de médio potencial (MP), e apenas 02 compostos, possuíam baixo potencial de transporte dissolvido em água.

Quando analisado o resultado de contaminação das águas superficiais nos dois meios de transporte (PTAS e PTDA), foi possível identificar que os princípios ativos: MSMA e Paraquate estiveram mais susceptíveis aos dois meios de transportes, logo se enquadraram na classe de alto risco, ou seja, maiores tendências de serem transportados e, probabilidade de contaminarem as águas superficiais.

No estudo desenvolvido por Milhome ${ }^{23}$ referente à análise do potencial de contaminação das águas superficiais dos agrotóxicos utilizados na agricultura do Perímetro Irrigado Jaguaribe -Apodi-CE, observou-se que, para a análise do 
Quadro 1. Distribuição dos princípios ativos utilizados na área em estudo quanto ao risco de contaminação em água superficial pelo método de GOSS.

\begin{tabular}{|l|l|l|}
\hline \multicolumn{2}{|c|}{ Análise do Risco de Contaminação em Água Superficial pelo Método de GOSS } \\
\hline & Risco & Princípio Ativo \\
\hline Associado ao Sedimento & Alto Potencial & MSMA e Paraquate \\
\cline { 2 - 3 } & Médio Potencial & $\begin{array}{l}\text { Glifosato; Fipronil; Fluazifope-P-Butílico; Clomazone; Lambda } \\
\text { Cialotrina; Imazapique; } \\
\text { Tebutiurom; Sulfentrazona; Oxyfluorfem; Diuron; } \\
\text { Hexazinona; Picloram; Imidacloprido; Flumioxazina. }\end{array}$ \\
\cline { 3 - 4 } & \multirow{2}{*}{ Baixo Potencial } & $\begin{array}{l}\text { Ametrina; Sulfometuron-Methyl; 2,4 D; Metribuzim; } \\
\text { Mesotriona; Isoxaflutol; Amicarbazona. }\end{array}$ \\
\cline { 3 - 4 } & Alto Potencial & $\begin{array}{l}\text { Ametrina; MSMA; Fipronil; Paraquate; Clomazone; } \\
\text { Imazapique, Tebutiurom; Sulfentrazona; } \\
\text { Diuron; Hexazinona; Picloram; Imidacloprido }\end{array}$ \\
\cline { 2 - 3 } & Médio Potencial & $\begin{array}{l}\text { Glifosato; Sulfometuron-Methyl; Fluazifope-P-Butílico; } \\
\text { 2,4-D; Metribuzin; Mesotriona; Isoxaflutol; Amicarbazona; } \\
\text { Flumioxazina. }\end{array}$ \\
\cline { 2 - 3 } & & Lambda-Cialotrina e Oxyfluorfem, \\
\cline { 2 - 3 } & Baixo Potencial & \\
\hline
\end{tabular}

Fonte: Autoras (2016).

PTAS a maioria dos compostos estudados encontrava-se inserida nas classes de risco: médio e baixo potencial de contaminação, e na análise do PTDA, a maior parcela dos princípios ativos classifica-se como de médio e alto potencial de contaminação, ou seja, a maioria dos compostos investigados possuíam uma maior tendência de transporte dissolvidos em água, o que corrobora com os resultados obtidos nesta pesquisa.

Os mesmos resultados de tendência de contaminação por ambos os meios de transportes foram encontrados por Gama et al. ${ }^{24}$ ao avaliarem os pesticidas utilizados na região Semiárida do Estado do Ceará, e por Martini et al. ${ }^{35}$ ao avaliarem o padrão de contaminação das águas por pesticidas recomendados para a cultura do arroz no Estado do Rio Grande do Sul.

\section{Considerações finais}

O levantamento dos agrotóxicos utilizados na cultura de cana-de-açúcar da usina mostrou que estão sendo lançados na área de estudo 25 princípios ativos em diferentes fases do ciclo vegetativo da cultura de cana-de-açúcar a fim de minimizar as perdas na produção e atender a demanda de mercado.
A avaliação do potencial de contaminação das águas subterrâneas evidenciou que a maioria dos princípios ativos investigados foram enquadrados na classe de risco dos contaminantes potenciais (CP). Quando comparado à tendência de contaminação das águas superficiais a presente pesquisa concluiu que a maior parcela dos princípios ativos analisados apresentou condições favoráveis de serem mais facilmente transportados por meio do escoamento superficial quando dissolvidos em água.

As informações obtidas nesta pesquisa sobre os possíveis impactos do uso de agrotóxicos nas águas subterrâneas e superficiais enfatizam a necessidade de implantação de programas de monitoramento ambiental priorizando os agrotóxicos potencialmente causadores de contaminação ambiental na área de estudo e em demais áreas sucroalcooleiras que fazem uso destes compostos. Além disso, o respectivo estudo é de grande importância na adoção de medidas preventivas voltadas a proteção da saúde pública principalmente para a população que está em contato direto com os agrotóxicos, bem como dos que fazem uso dos recursos hídricos da área. 


\section{Colaboradores}

LL Ismael: responsável pela escrita, pesquisa, coleta e análise dos resultados e formatação do manuscrito. EMR Rocha: responsável pela coleta, análise dos dados e correções do manuscrito.

\section{Referências}

1. Veiga MM. Agrotóxicos: eficiência econômica e injustiça socioambiental. Cien Saude Colet 2005; 12(1):145-152.

2. Ferreira MJM, Viana Júnior MM, Pontes AGV, Rigotto, RM, Gadelha D. Gestão e uso dos recursos hídricos e a expansão do agronegócio: água para quê e para quem? Cien Saude Colet 2016; 21(3):743-752

3. Domingues E. Sistema de informação geográfica e a contaminação de agrotóxicos no meio ambiente da cana-de-açúcar na bacia hidrográfica do Rio Pardo - SP/ $M G$ [tese]. São Paulo: Universidade Estadual Paulista; 2010.

4. Sinha SN, Bhatnagar VK, Doctor P, Toteja GS, Agnihotri NP, Kalra RL. A Novel Method for Pesticide Analysis in Refined Sugar Samples Using a Gas Chromatography-Mass Spectrometer (GC-MS/MS) and Simple Solvent Extraction Method. Food Chemistry 2011; 126(1):379-386.

5. Oliveira TG, Favareto APA, Antunes PA. Agrotóxicos: levantamento dos mais utilizados no oeste Paulista e seus efeitos como desreguladores endócrinos. Fórum Ambiental da Alta Paulista 2013; 9(11):375-390

6. Silva JF. Degradação Ambiental a partir da Cultura da Cana-de-Açúcar, no Município de Itapororoca - PB [dissertação]. Guarabira: Universidade Estadual da Paraíba; 2010.

7. Matias J. Nova expansão canavieira, mudanças espaciais e produtivas: o caso do município de Santa Rita - $P B$ [dissertação]. João Pessoa: Universidade Federal da Paraíba; 2010.

8. Figueiredo TQ. A produção sucroalcooleira e a sustentabilidade: um diagnóstico do início do século XXI na Paraíba. Olhares Plurais 2010; 1(2):88-104.

9. Sistema Nacional de Informações Tóxico-Farmacológicas (Sinitox). Casos de intoxicações e morte por agrotóxicos de uso agrícola registrados na Paraíba. Rio de Janeiro: Sinitox; 2016.

10. Moreira JC, Jacob SC, Peres F, Lima JS, Meyer A, Oliveira-Silva JJ, Sarcinelli PN, Batista DF, Egler M, Faria MVC, Araújo AJ, Kubota AH, Soares MO, Alves SR, Moura CM, Curi R. Avaliação integrada do impacto do uso de agrotóxicos sobre a saúde humana em uma comunidade agrícola de Nova Friburgo, RJ. Cien Saude Colet 2002; 7(2):299-311.

11. Palma DCA, Lourencetti C. Agrotóxicos em água e alimentos: Riscos à saúde humana. Revis Uniara 2011; 14(2):7-21.

12. Arias ARL, Buss DF, Alburquerque C, Inácio AF, Freire MM, Egler M, Mugnai R, Baptista DF. Utilização de bioindicadores na avaliação de impacto e no monitoramento da contaminação de rios e córregos por agrotóxicos. Cien Saude Colet 2007; 12(1):61-72.

13. Spadotto CA. Abordagem interdisciplinar na avaliação ambiental de agrotóxicos. Núcleo de Pesq Inter 2006; [acessado 2016 Jul 24]. Disponível em: http://www. academia.edu/12466251/ABORDAGEM_INTERDISCIPLINAR_NA_AVALIA\%C3\%87\%C3\%83O_AMBIENTAL_DE_AGROT\%C3\%93XICOS 
14. Silva CL. Análise da vulnerabilidade ambiental aos principais pesticidas recomendados para os Sistemas de produção de algodão, arroz, café, cana-de-açúcar, citros, milho e soja [dissertação]. Campinas: Universidade Estadual de Campinas; 2004.

15. Milhome MAL, Sousa DOB, Lima FAF, Nascimento RF. Avaliação do potencial de contaminação de águas superficiais e subterrâneas por pesticidas aplicados na agricultura do Baixo Jaguaribe, CE. Engen Sanit e Ambi 2009; 14(3):363-372.

16. Britto FB. Pesticidas no alto do Rio Poxim e os riscos de contaminação [dissertação]. São Cristovão: Universidade Federal do Sergipe; 2011.

17. Rocha AA. Monitoramento de agrotóxicos em áreas ir rigadas por pivô central na microbacia do Tijunqueiro, município de Morrinhos, Goiás [tese]. Piracicaba: Universidade de São Paulo; 2011.

18. Fernandes Neto ML, Sarcinelli PN. Agrotóxicos em água para consumo humano: uma abordagem de avaliação de risco e contribuição ao processo de atualização da legislação brasileira. Eng Sanit e Ambi 2009; 14(1):69-78.

19. Brasil. Portaria $\mathrm{n}^{\circ} 2.914$, de 2 de dezembro de 2011 . Dispõe sobre os procedimentos de controle e de vigilância da qualidade da água para consumo humano e seu padrão de potabilidade. Diário Oficial da União 2011; 2 set.

20. Associação Brasileira de Saúde Coletiva (Abrasco). Um alerta sobre os impactos dos agrotóxicos na saúde. Dossiê ABRASCO. Parte 1 - Agrotóxicos, segurança alimentar e nutricional e saúde. Rio de Janeiro: Abrasco; 2012.

21. Armas ED, Monteiro RTR. Uso de agrotóxicos em cana-de-açúcar na bacia do rio Corumbataí e o risco de poluição hídrica. Quimica Nova 2005; 28(6):975-982.

22. Marques MN, Badiru AI, Beltrame O, Pires MAF. Pesticide Leaching and Run-off Hazard in the Ribeira de Iguape River Basin in São Paulo State, Brazil. Jorn. Braz. Soc. Ecotoxicol 2007; 2(2):179-185.

23. Milhome MAL. Influência do uso de agrotóxicos na qualidade dos recursos hídricos da região do Perímetro Irrigado Jaguaribe-Apodi/CE [tese]. Fortaleza: Universidade Federal do Ceará; 2011.

24. Gama AF, Oliveira AHB, Cavalcante RM. Inventário de agrotóxicos e risco de contaminação química dos recursos hídricos no Semiárido Cearense. Química Nova 2013; 36(3):462-467.

25. Appolinário F. Metodologia da Ciência: filosofia e prática da pesquisa. São Paulo: Pioneira Thomson Learning; 2006.

26. Kauark FS, Mahães FC, Medeiros AC. Metodologia da Pesquisa: Um guia prático. Itabuna: Via Litterarum; 2010.

27. Gil AC. Como Elaborar Projetos de Pesquisa. $4^{\text {a }}$ ed. São Paulo: Editora Atlas S.A.; 2002.

28. Agência de Defesa Agropecuária do Paraná (Adapar). Pesquisa de Bulas de Agrotóxicos. Curitiba: Adapar. [acessado 2016 Jan 15]. Disponível em: http://www. adapar.pr.gov.br/modules/conteudo/conteudo.php?conteudo $=389$.
29. Agência Nacional de Vigilância Sanitária (Anvisa). Regularização de produtos - Agrotóxicos/ Monografias autorizadas. [acessado 2016 Mar 10]. Disponível em: http://portal.anvisa.gov.br/registros-e-autorizacoes/ agrotoxicos/produtos/monografia-de-agrotoxicos/ autorizadas

30. The Footprint (PPDB). Pesticide properties database. Hertfordshire: University of Hertfordshire; 2015.

31. Andrade AS, Queiroz VT, Lima DT, Drumond LCD. Análise de risco de contaminação de águas superficiais e subterrâneas por pesticidas em municípios do Alto Paranaíba - MG. Química Nova 2011; 34(7):11291135.

32. Gustafson DI. Groundwater ubiquity score: a simple method for assessing pesticide leachability. Environmental Toxicology Chemicals 1989; 8:339.

33. Goss DW. Screening procedure for soils and pesticides for potential water quality impacts. Weed Technol 1992; 6:701.

34. Marques MN. Avaliação do impacto de agrotóxico e áreas de proteção ambiental pertencente à bacia hidrográfica do Rio Ribeira de Iguape São Paulo: uma contribuição à análise crítica da legislação sobre o padrão de potabilidade [tese]. São Paulo: Instituto de Pesquisas Energéticas e Nucleares; 2005.

35. Martini LFD, Caldas SS, Bolzan CM, Bundt ADC, Primel EG, Avila LA. Risco de contaminação das águas de superfície e subterrâneas por agrotóxicos recomendados para a cultura do arroz irrigado. Ciência Rural 2012; 42(10):1715-1721.

36. Ruy R, Reis TES. Risco de contaminação por agrotóxicos das águas subterrâneas em áreas cultivadas com cana-de-açúcar. Pesti: Revis Ecotox e Meio Amb 2012; 22:77-84.

37. Primel EG, Zanella R, Kurz MHS, Gonçalves FF, Machado SO, Marchezan E. Poluição das águas por herbicidas utilizados no cultivo do arroz irrigado na região central do estado do Rio Grande do Sul, Brasil: predição teórica e monitoramento. Química Nova 2005; 28(4):605-609.

Artigo apresentado em 01/11/2017

Aprovado em 03/05/2018

Versão final apresentada em 05/05/2018 
